\title{
Convexity of Decentralized Controller Synthesis
}

\author{
Laurent Lessard Sanjay Lall
}

\begin{abstract}
In decentralized control problems, a standard approach is to specify the set of allowable decentralized controllers as a closed subspace of linear operators. This then induces a corresponding set of Youla parameters. Previous work has shown that quadratic invariance of the controller set implies that the set of Youla parameters is convex. In this paper, we prove the converse. We thereby show that the only decentralized control problems for which the set of Youla parameters is convex are those which are quadratically invariant. We further show that under additional assumptions, quadratic invariance is necessary and sufficient for the set of achievable closed-loop maps to be convex. We give two versions of our results. The first applies to bounded linear operators on a Banach space and the second applies to (possibly unstable) causal LTI systems in discrete or continuous time.
\end{abstract}

\section{Introduction}

This paper considers the feedback control of linear systems subject to structural constraints on the controller. We are interested in characterizing when the set of achievable closed-loop maps is convex. Convexity is important because in many cases it makes the problem of synthesizing structurally constrained controllers that optimize some performance objective a tractable one.

Suppose $w \in \mathcal{W}$ is the exogenous disturbance, $u \in \mathcal{U}$ is the control input, $z \in \mathcal{Z}$ is the regulated output, and $y \in \mathcal{Y}$ is the measurement. We assume that the plant is a linear and continuous map $P:(\mathcal{W}, \mathcal{U}) \rightarrow(\mathcal{Z}, \mathcal{Y})$. The controller is a map $K: \mathcal{Y} \rightarrow \mathcal{U}$ that is connected to the plant in feedback. We partition the four blocks of $P$ as $P_{i j}$ and by convention we let $G=P_{22}$. The closed-loop interconnection mapping $w \mapsto z$ is depicted in Figure 1.

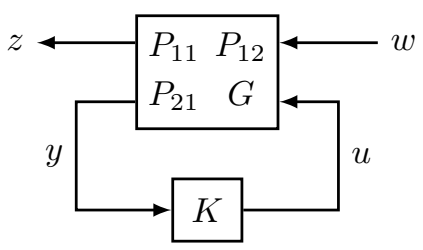

Figure 1: Plant $P$ connected to a controller $K$.

The set of achievable closed-loop maps is

$$
C=\left\{P_{11}+P_{12} K(I-G K)^{-1} P_{21} \mid K \in S\right\}
$$

Here the set $S$ is the set of $K$ satisfying the structural constraints imposed in the problem. Examples of such structural constraints include sparsity requirements, where for example the controller responsible for choosing $u_{j}$ cannot measure $y_{i}$. Other possibilities include constraints arising as a consequence of measurement delays. To make notation more compact, we define the function

$$
h(K)=-K(I-G K)^{-1}
$$

and then we may write $C=P_{11}-P_{12} h(S) P_{21}$ where $h(S)$ is the image of $S$ under the map $h$. Note that $h$ implicitly depends on the map $G$. In this paper we refer to $h$ and $G$ together with the understanding that $h$ is defined as a function of $G$. A constrained synthesis problem may take the form

$$
\begin{aligned}
\operatorname{minimize} & \|X\| \\
\text { subject to } & X \in C
\end{aligned}
$$

where $\|\cdot\|$ is some convex measure of performance, such as a norm. It is desirable to know when $C$ is a convex set, because then if (2) is a convex optimization problem.

A set $S$ is called quadratically invariant under $G$ if $K G K \in S$ for all $K \in S$. It is shown in [12,13] in that, roughly speaking, $h(S)=S$ if and only if $S$ is quadratically invariant under $G$. This provides a sufficient condition under which $C$ is convex.

This work builds on the notion of quadratic invariance developed in $[12,13]$. This earlier work showed that quadratic invariance is sufficient for $h(S)$ and $C$ to be convex. In this paper, our main contributions are as follows.

(i) We show that quadratic invariance is a necessary condition for convexity of $h(S)$.

(ii) We show that, subject to some additional assumptions, quadratic invariance is also a necessary condition for convexity of $C$.

(iii) We give examples that illustrate both the generality of the results as well as the limitation encountered when the additional assumptions in (ii) are violated.

The paper is organized as follows. We begin by reviewing related works in the literature and covering necessary mathematical preliminaries, including a review of quadratic invariance. In Section III we prove our main results and in Section IV we show some illustrative examples. Finally, we conclude in Section V. 


\section{I-A Prior work}

Optimal controller synthesis subject to information constraints is known to be hard in general [2]. Even when the plant is linear, the noise is Gaussian, and the cost function is quadratic, the optimal controller may not be linear [18]. Furthermore, there is currently no known efficient algorithm for finding the optimal linear controller in general.

Despite these difficulties, the pervasiveness of decentralized information in large-scale systems has driven researchers to seek subclasses of problems that are tractable. Early work by Radner [11] showed that some static team decision problems admit optimal controllers that are linear. This was extended to dynamic teams by Ho and Chu [3] under the assumption that the information structure is partially nested.

More recently, many works have addressed the broad area of decentralized control synthesis, including $[8,9$, 14,17]. Specific efforts have focused on characterizing when the set of achievable closed-loop maps as detailed in Section I is convex. For example, $[10,15]$ shows several classes of information constraints that lead to a convex $C$. In [13] it is shown that the simple algebraic condition of quadratic invariance encompasses a wide class of problems for which $C$ is convex.

However, quadratic invariance is only a sufficient condition for convexity of $C$. Recent work $[5,6]$ introduces the concept of internal quadratic invariance, which gives a more complete characterization of $C$. This condition highlights cases where quadratic invariance does not hold, but a suitable transformation can produce a new problem for which $C$ is unchanged and quadratic invariance now holds.

One may also characterize $C$ more directly. Using tools from algebraic geometry, specifically elimination theory, the paper [16] gives a method for computing a representation of the smallest algebraic variety containing $C$. This does not necessarily make the problem of verifying convexity easier, but it does open the door to other tools for identifying convexity such as sum-of-squares relaxations. See [1] and references therein.

This paper gives conditions under which quadratic invariance is both necessary and sufficient for convexity. Preliminary versions of some of these results appeared in $[4,7]$ for the Banach space case only. In this paper, we include new results that cover the extended spaces $\ell_{2 e}$ and $L_{2 e}$, and we present new examples in Section IV that illustrate both the generality and limitations of our results.

\section{Preliminaries}

If $\mathcal{X}$ and $\mathcal{Y}$ are topological vector spaces (TVS), we let $\mathcal{L}(\mathcal{X}, \mathcal{Y})$ denote the set of all maps $T: \mathcal{X} \rightarrow \mathcal{Y}$ such that $T$ is linear and continuous. This paper concerns properties of linear operators. What we are able to prove depends on the underlying structure of the vector spaces involved, so we distinguish two classes of topological vector spaces: Banach spaces, and the extended spaces $\ell_{2 e}$ and $L_{2 e}$.

\section{Banach spaces}

A Banach space is a TVS whose topology is induced by a norm, and the space is complete. Any linear and continuous map from one Banach space to another is bounded. Common examples of Banach spaces include $\ell_{p}$ and $L_{p}$; the set of functions $f: \mathbb{Z}_{+} \rightarrow \mathbb{R}$ and Lebesguemeasurable functions $f: \mathbb{R}_{+} \rightarrow \mathbb{R}$ respectively, for which the $p$-norm is finite. Simpler examples include the Hilbert spaces $\mathbb{R}^{n}, \ell_{2}$, or $L_{2}$. Suppose $\mathcal{U}$ and $\mathcal{Y}$ are Banach spaces, and $G \in \mathcal{L}(\mathcal{U}, \mathcal{Y})$. Define the following set.

$$
M=\{K \in \mathcal{L}(\mathcal{Y}, \mathcal{U}) \mid I-G K \text { is invertible }\}
$$

Note that the set $M$ is precisely the domain of $h$. For any $A \in \mathcal{L}(\mathcal{Y}, \mathcal{Y})$, the resolvent set is defined as $\rho(A)=$ $\{\lambda \in \mathbb{C} \mid(\lambda I-A)$ is invertible $\}$. It is a fact that $\rho(A)$ is always an open set, and contains all $\lambda \in \mathbb{C}$ for which $|\lambda|>$ $\|A\|$. Therefore, one may define $\rho_{\text {uc }}(A)$, the unbounded connected component of $\rho(A)$. Now define the subset $N \subseteq M$ as follows.

$$
N=\left\{K \in \mathcal{L}(\mathcal{Y}, \mathcal{U}) \mid 1 \in \rho_{\mathrm{uc}}(G K)\right\}
$$

\section{Extended spaces}

Banach spaces are well-suited for representing a wide variety of systems, but when the time horizon is infinite, such as with $\ell_{2}$ and $L_{2}$, only bounded maps are permitted. In order to represent unbounded maps as well, such as unstable systems, we use the notion of extended spaces. First, define the truncation and shift operators $P_{T}$ and $D_{T}$ which operate on functions $f: \mathbb{Z}_{+} \rightarrow \mathbb{R}$ or $f: \mathbb{R}_{+} \rightarrow \mathbb{R}$, as follows. For $T>0$, define

$$
P_{T} f= \begin{cases}f(t) & \text { if } t \leq T \\ 0 & \text { otherwise }\end{cases}
$$

and

$$
D_{T} f= \begin{cases}0 & \text { if } t<T \\ f(t-T) & \text { otherwise }\end{cases}
$$

The extended spaces $\ell_{p e}$ and $L_{p e}$ are defined as follows.

$$
\begin{aligned}
\ell_{p e} & =\left\{f: \mathbb{Z}_{+} \rightarrow \mathbb{R} \mid P_{T} f \in \ell_{p} \text { for all } T \in \mathbb{Z}_{+}\right\} \\
L_{p e} & =\left\{f: \mathbb{R}_{+} \rightarrow \mathbb{R} \mid P_{T} f \in L_{p} \text { for all } T \in \mathbb{R}_{+}\right\}
\end{aligned}
$$

Note that $\ell_{p e}$ is the same for every $p$, and is the set of real sequences, which we abbreviate as $\ell_{e}$. However, $L_{p e}$ is the set of functions that are $L_{p}$ on finite intervals, and so is different for different $p$. 
A map $A \in \mathcal{L}\left(L_{2 e}^{m}, L_{2 e}^{n}\right)$ or $A \in \mathcal{L}\left(\ell_{2 e}^{m}, \ell_{2 e}^{n}\right)$ is called causal if, for all $T \geq 0$, we have $P_{T} A P_{T}=P_{T} A$. A causal system $A$ is called time-invariant if $D_{T} A=A D_{T}$ for all $T>0$. We denote the set of linear, causal, timeinvariant maps as $\mathcal{L}_{\mathrm{TI}}$.

The extended spaces are not Banach spaces, but we may endow them with a suitable topology and recover notions of convergence and continuity. Let the topology on $L_{2 e}$ be generated by all the open $\|\cdot\|_{T}$-balls for every $T \in \mathbb{R}_{+}$, where we define $\|f\|_{T}=\left\|P_{T} f\right\|_{L_{2}}$. Also, we let the topology on $\mathcal{L}\left(L_{2 e}^{m}, L_{2 e}^{n}\right)$ be generated by all the open $\|\cdot\|_{T}$-balls for every $T \in \mathbb{R}_{+}$, where $\|A\|_{T}=\left\|P_{T} A\right\|_{L_{2}^{m} \rightarrow L_{2}^{n}}$. It can be shown that these topologies are Hausdorff, and thus $L_{2 e}$ and $\mathcal{L}\left(L_{2 e}^{m}, L_{2 e}^{n}\right)$ are topological vector spaces (TVS). Furthermore, convergence in this topology is equivalent to convergence in every $\|\cdot\|_{T}$-norm, and continuity of a linear operator in this topology is equivalent to continuity in every $\|\cdot\|_{T^{-}}$ norm. A similar topology is defined for $\ell_{2 e}$. For a thorough treatment of these concepts, see for example [20]. We also require the concept of an inert subspace, which we give below.

Definition 1. The set $S \subseteq \mathcal{L}_{\mathrm{TI}}\left(L_{2 e}^{n_{u}}, L_{2 e}^{n_{y}}\right)$ is inert with respect to $G \in \mathcal{L}_{\mathrm{TI}}\left(L_{2 e}^{n_{y}}, L_{2 e}^{n_{u}}\right)$ if for all $K \in S,(g k)_{i j} \in$ $L_{\infty e}$ for all $i, j=1, \ldots, n_{y}$ where $(g k)$ is the impulse response matrix of $G K$. We overload our notation and also define $S \subseteq \mathcal{L}_{\mathrm{TI}}\left(\ell_{2 e}^{n_{u}}, \ell_{2 e}^{n_{y}}\right)$ to be inert with respect to $G \in \mathcal{L}_{\mathrm{TI}}\left(\ell_{2 e}^{n_{u}}, \ell_{2 e}^{n_{y}}\right)$ if for all $K \in S,(g k)_{i j} \in \ell_{e}$ for all $i, j=1, \ldots, n_{y}$ and $r((g k)(0))<1$ where $(g k)$ is the discrete impulse response matrix of $G K$ and $r(\cdot)$ denotes the spectral radius.

Note in particular that if $S$ is inert with respect to $G$, then $I-G K$ is invertible for all $K \in S$. A proof of this result is in [13].

\section{Quadratic invariance}

We now summarize the definitions and main results regarding quadratic invariance. The following definition may be found in $[12,13]$.

Definition 2. Suppose $\mathcal{U}$ and $\mathcal{Y}$ are TVS. Suppose $S \subseteq$ $\mathcal{L}(\mathcal{Y}, \mathcal{U})$ and $G \in \mathcal{L}(\mathcal{U}, \mathcal{Y})$. The set $S$ is quadratically invariant under $G$ if $K G K \in S$ for all $K \in S$.

The first quadratic invariance result we present applies to Banach spaces.

Theorem 3 (from [12]). Suppose $\mathcal{U}$ and $\mathcal{Y}$ are Banach spaces. Suppose $S \subseteq \mathcal{L}(\mathcal{Y}, \mathcal{U})$ is a closed subspace and $G \in \mathcal{L}(\mathcal{U}, \mathcal{Y})$. Define $M$ and $N$ as in (3)-(4), and suppose $S \cap N=S \cap M$. Then $S$ is quadratically invariant under $G$ if and only if $h(S \cap M)=S \cap M$.

The second quadratic invariance result applies to the topological vector spaces $\ell_{2 e}$ and $L_{2 e}$.
Theorem 4 (from [13]). Suppose $S \subseteq \mathcal{L}_{\mathrm{TI}}\left(L_{2 e}^{n_{u}}, L_{2 e}^{n_{y}}\right)$ is an inert closed subspace and $G \in \overline{\mathcal{L}}_{\mathrm{TI}}\left(L_{2 e}^{n_{u}}, L_{2 e}^{n_{y}}\right)$, or suppose $S \subseteq \mathcal{L}_{\mathrm{TI}}\left(\ell_{2 e}^{n_{u}}, \ell_{2 e}^{n_{y}}\right)$ is an inert closed subspace and $G \in \mathcal{L}_{\mathrm{TI}}\left(\ell_{2 e}^{n_{u}}, \ell_{2 e}^{n_{y}}\right)$. Then $S$ is quadratically invariant under $G$ if and only if $h(S)=S$.

Roughly speaking, $S$ being quadratically invariant under $G$ means that the set $S$ is invariant under the application of $h$. Technical conditions arise only to ensure that we avoid $K$ for which $h(K)$ is not well-defined. In the case of Theorem 3, this amounts to intersecting $S$ with $M$, the domain of $h$. In Theorem 4, we make the technical assumption that $S$ is an inert subspace, and this ensures that $h(K)$ is always well-defined.

\section{Main results}

We begin by defining two types of sets that will be useful in our main results. These definitions apply to any vector space over $\mathbb{R}$, so in particular they apply to both Banach spaces and the topological vector spaces $\ell_{2 e}$ and $L_{2 e}$.

Definition 5. Suppose $\mathcal{V}$ is a vector space over $\mathbb{R}$, and $S \subseteq \mathcal{V}$. We call $S$ homogeneous if for all $v \in S$ and $\alpha \in \mathbb{R}$, we have $\alpha v \in S$.

Homogeneous sets are collections of lines that pass through the origin. If a point belongs to a homogeneous set, so does the line that passes through that point and the origin. Note that every subspace is homogeneous, but not all homogeneous sets are subspaces.

Definition 6. Suppose $\mathcal{V}$ is a vector space over $\mathbb{R}$, and $T \subseteq \mathcal{V}$. We call $T$ star-shaped if for all $v \in T$ and $\alpha \in[0,1]$, we have $\alpha v \in T$.

If a point belongs to a homogeneous set, so does the line segment connecting it to the origin. Therefore, every homogeneous set is star-shaped, but not vice-versa. Furthermore, every convex set containing the origin is star-shaped, but not vice-versa. For examples of these sets in $\mathbb{R}^{2}$, see Figure 2 .

\section{III-A Banach space results}

Our first main result states that if $h$ maps a homogeneous subset of its domain to a star-shaped set, it must in fact map that homogeneous set to itself. We will see that this result has implications concerning convexity.

Theorem 7. Suppose $\mathcal{U}$ and $\mathcal{Y}$ are Banach spaces. Suppose $S \subseteq \mathcal{L}(\mathcal{Y}, \mathcal{U})$ is closed and homogeneous and $G \in$ $\mathcal{L}(\mathcal{U}, \mathcal{Y})$. Define $M$ and $N$ as in (3)-(4), and suppose $S \cap N=S \cap M$. If $h(S \cap M)=T \cap M$ for some starshaped set $T$, then $T \cap M=S \cap M$.

Proof. Suppose $h(S \cap M)=T \cap M$, where $T$ is a starshaped set. Fix some $K \in S \cap M$. Therefore, $I-G K$ 


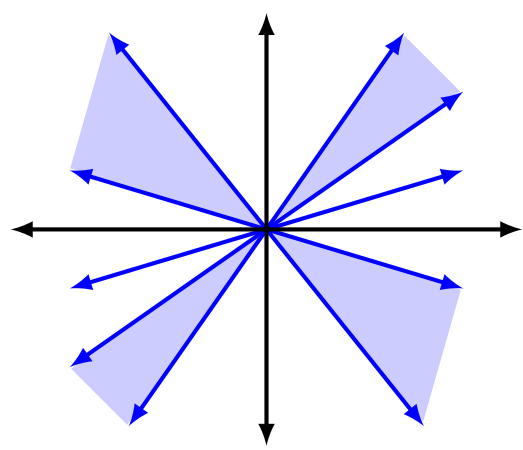

(a) homogeneous set

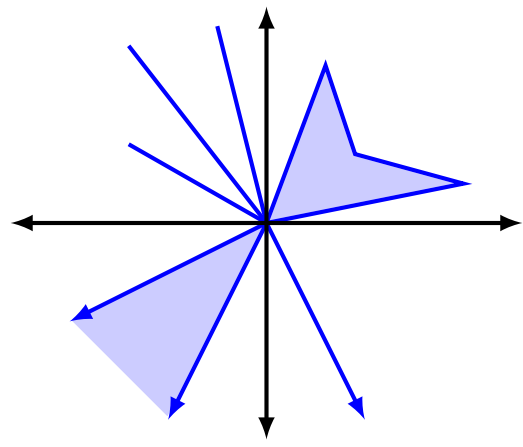

(b) star-shaped set

Figure 2: Example of a homogeneous set and a starshaped set in $\mathbb{R}^{2}$. Lines with arrows indicate that they extend to infinity.

is invertible, and $1 \in \rho(G K)$. The resolvent set of a bounded linear operator is an open set, so there exists a sufficiently small $\varepsilon \in(0,1]$ such that $1-\alpha \in \rho(G K)$ for all $\alpha \in[0, \varepsilon]$. For any such $\alpha$, it follows that $I-(1-\alpha) G K$ is invertible. Therefore, $(I-(1-\alpha) G K)(I-G K)^{-1}$ is invertible as well. Expanding this expression, we find that it equals $I-\alpha G h(K)$. Thus $\alpha h(K) \in M$.

Also, we have $h(K) \in T$ by assumption, and so $\alpha h(K) \in T$ whenever $\alpha \in[0,1]$, because $T$ is a starshaped set. It follows that for $\alpha \in[0, \varepsilon], \alpha h(K) \in T \cap M$. Applying $h$ to both sides, we conclude that $h(\alpha h(K)) \in$ $h(T \cap M)=S \cap M$, where we made use of the involutive property of $h$. Expanding $h(\alpha h(K))$, we find that it equals $\alpha K(I-(1-\alpha) G K)^{-1}$. Since $S$ is a homogeneous set, we may multiply this expression by $-1 / \alpha$ when $\alpha \neq 0$, and the result will still lie in $S$. Thus, $-K(I-(1-\alpha) G K)^{-1} \in S$.

Now define the function $g:[0, \varepsilon] \rightarrow \mathcal{L}(\mathcal{Y}, \mathcal{U})$ by

$$
g(\alpha)=-K(I-(1-\alpha) G K)^{-1}
$$

Notice that $g$ is continuous at 0 , since $I-(1-\alpha) G K$ is invertible for sufficiently small $\alpha \geq 0$ as above, and the inversion map is continuous on its domain. Since $S$ is closed and $g(\alpha) \in S$ for $\alpha \in(0, \varepsilon]$, we have

$$
\lim _{\alpha \rightarrow 0^{+}} g(\alpha) \in S
$$

We may take the limit $\alpha \rightarrow 0^{+}$by simply evaluating $g$ at $\alpha=0$. Thus, we conclude that $h(K) \in S$. Now $h$ is a bijection from $M$ to $M$, and so we actually have $h(K) \in S \cap M$. Since $K$ was an arbitrary element of $S \cap M$, it follows that $h(S \cap M) \subseteq S \cap M$. Using the involutive property of $h$ once more, $h(S \cap M)=S \cap M$, as required.

Theorem 7 may be specialized to the case where $S$ is a subspace, and combined with Theorem 3 to yield a necessary condition for convexity of $h$.

Corollary 8. Suppose $\mathcal{U}$ and $\mathcal{Y}$ are Banach spaces. Suppose $S \subseteq \mathcal{L}(\mathcal{Y}, \mathcal{U})$ is a closed subspace and $G \in \mathcal{L}(\mathcal{U}, \mathcal{Y})$. Define $M$ and $N$ as in (3)-(4), and suppose $S \cap N=$ $S \cap M$. Then the following statements are equivalent.

(i) $S$ is quadratically invariant under $G$

(ii) $h(S \cap M)=S \cap M$

(iii) $h(S \cap M)=\Gamma \cap M$ for some convex set $\Gamma$

Proof. (i) $\Longleftrightarrow$ (ii) is precisely Theorem 3 . The case (ii) $\Longrightarrow$ (iii) is immediate. Finally, to show (ii) $\Longleftarrow(i i i)$, notice that if (iii) holds then $\Gamma$ must contain the origin, and since it is convex it must therefore be star-shaped. Then, since every subspace is homogeneous, (ii) follows from Theorem 7 .

Corollary 8 shows that quadratic invariance is necessary and sufficient for convexity of $h$. We now show that under additional invertibility assumptions, this result also applies to the set of achievable closed-loop maps described in Section I.

Corollary 9. Suppose the conditions of Corollary 8 hold. Additionally, suppose $\mathcal{W}$ and $\mathcal{Z}$ are Banach spaces, and $P_{11} \in \mathcal{L}(\mathcal{W}, \mathcal{Z}), P_{12} \in \mathcal{L}(\mathcal{U}, \mathcal{Z})$, and $P_{21} \in \mathcal{L}(\mathcal{W}, \mathcal{Y})$ Finally, suppose $S \cap M=S$. If $P_{12}$ is left-invertible and $P_{21}$ is right-invertible, then the following statements are equivalent.

(i) $S$ is quadratically invariant under $G$

(ii) $P_{11}-P_{12} h(S) P_{21}=\Gamma$, where $\Gamma$ is a convex set.

Proof. The proof of $(i) \Longrightarrow$ (ii) follows directly from Theorem 7. Conversely, suppose that (ii) holds. Let $P_{12}^{\dagger}$ be a left-inverse of $P_{12}$ and let $P_{21}^{\dagger}$ be a right-inverse of $P_{21}$. Then we have

$$
h(S)=P_{12}^{\dagger} P_{11} P_{21}^{\dagger}-P_{12}^{\dagger} \Gamma P_{21}^{\dagger}
$$

where the right-hand side is a convex set, because it is an affine transformation of the convex set $\Gamma$. Applying Corollary 8 , we conclude that $S$ is quadratically invariant under $G$, as required.

In Section IV, we will show some examples that illustrate why the invertibility requirements are necessary in this result. 


\section{III-B Extended space results}

We now present results analogous to those of Section III-A, but now for the extended spaces $\ell_{2 e}$ and $L_{2 e}$.

Theorem 10. Suppose $S \subseteq \mathcal{L}_{\mathrm{TI}}\left(L_{2 e}^{n_{y}}, L_{2 e}^{n_{u}}\right)$ is inert, closed, and homogeneous and $G \in \mathcal{L}_{\mathrm{TI}}\left(L_{2 e}^{n_{y}}, L_{2 e}^{n_{u}}\right)$. If $h(S)=T$ where $T$ is a star-shaped set, then $T=S$. This result also holds when $L_{2 e}$ is replaced by $\ell_{2 e}$.

Proof. This proof is similar to that of Theorem 7, except we do not need to worry about the invertibility of $I-$ $G K$, since it is guaranteed by the inertness property of $S$. Suppose $h(S)=T$, where $T$ is a star-shaped set. Applying $h$ to both sides, we conclude that $h(T)=S$. Together with the star-shaped property of $T$, it follows that $h(\alpha h(S)) \subseteq S$ for any $\alpha \in[0,1]$. As in the proof of Theorem 7, we conclude that $g(\alpha) \in S$ for all $\alpha \in(0,1]$, where $g$ is defined in (5). The rest of the proof follows as in the proof of Theorem 7 . The only difference is that we must verify continuity of $g$ at 0 using the topology defined in Section II.

As in Section III-A, Theorem 10 may be specialized to the case where $S$ is a subspace, and combined with Theorem 4 to yield a necessary condition for convexity of $h$.

Corollary 11. Suppose $S \subseteq \mathcal{L}_{\mathrm{TI}}\left(L_{2 e}^{n_{y}}, L_{2 e}^{n_{u}}\right)$ is an inert and closed subspace and $G \in \mathcal{L}_{\mathrm{TI}}\left(L_{2 e}^{n_{y}}, L_{2 e}^{n_{u}}\right)$. Then the following statements are equivalent

(i) $S$ is quadratically invariant under $G$

(ii) $h(S)=S$

(iii) $h(S)$ is convex

This result also holds when $L_{2 e}$ is replaced by $\ell_{2 e}$.

Proof. See the proof of Corollary 8 .

Corollary 11 shows that quadratic invariance is necessary and sufficient for convexity of $h$. As in Section III-A, this result also applies to the set of achievable closed-loop maps when we make additional invertibility assumptions.

Corollary 12. Suppose the conditions of Corollary 11 hold, and $P \in \mathcal{L}_{\mathrm{TI}}\left(L_{2 e}, L_{2 e}\right)$. If $P_{12}$ is left-invertible and $P_{21}$ is right-invertible, then the following statements are equivalent

(i) $S$ is quadratically invariant under $G$

(ii) $P_{11}-P_{12} h(S) P_{21}$ is convex

This result also holds when $L_{2 e}$ is replaced by $\ell_{2 e}$.

Proof. See the proof of Corollary 9.

\section{Examples}

\section{LQG with sparsity}

Consider the extended space of signals $L_{2 e}$, and suppose we have the LTI map

$$
\left[\begin{array}{cc}
P_{11} & P_{12} \\
P_{21} & G
\end{array}\right]:\left[\begin{array}{c}
L_{2 e}^{n_{w}} \\
L_{2 e}^{n_{u}}
\end{array}\right] \rightarrow\left[\begin{array}{c}
L_{2 e}^{n_{z}} \\
L_{2 e}^{n_{y}}
\end{array}\right]
$$

given by the following minimal state-space realization.

$$
\left[\begin{array}{cc}
P_{11} & P_{12} \\
P_{21} & G
\end{array}\right]=\left[\begin{array}{c|cc}
A & B_{1} & B_{2} \\
\hline C_{1} & 0 & D_{12} \\
C_{2} & D_{21} & 0
\end{array}\right]
$$

We now make the classical assumptions typically made in $\mathcal{H}_{2}$ synthesis. These assumptions ensure that the controller that achieves a closed-loop map with minimum norm exists, is unique, and is rational [19]. They are as follows.

(i) $\left(C_{2}, A, B_{2}\right)$ is stabilizable and detectable

(ii) For all $\omega \in \mathbb{R}$ the matrices $\left[\begin{array}{cc}A-j \omega I & B_{2} \\ C_{1} & D_{12}\end{array}\right]$ and $D_{12}$ have full column rank

(iii) For all $\omega \in \mathbb{R}$ the matrices $\left[\begin{array}{cc}A-j \omega I & B_{1} \\ C_{2} & D_{21}\end{array}\right]$ and $D_{21}$ have full row rank

Now suppose we seek a controller $K \in \mathcal{L}_{\mathrm{TI}}\left(L_{2 e}^{n_{y}}, L_{2 e}^{n_{u}}\right)$ where $K$ has some prescribed sparsity structure. So $K \in S$ for some closed subspace $S$. Assumptions (ii) and (iii) ensure that $P_{12}$ is left-invertible and $P_{21}$ is rightinvertible, respectively. Therefore, we may apply Corollary 12 and conclude that quadratic invariance is necessary and sufficient for convexity of the set of achievable closed-loop maps. Thus for this class of problems, quadratic invariance may be tested to determine convexity. In particular, if $S$ is defined as the set of transfer functions with desired sparsity or delays, then quadratic invariance may be computationally tested using the methods in [13].

It is worth noting that under the above assumptions there are only two possibilities. Either $S$ is quadratically invariant, in which case the set of closed-loop maps $C$ must be given by $C=P_{11}-P_{12} S P_{21}$, or $S$ is not quadratically invariant in which case $C$ is not convex. Additionally, one can conclude structural properties. For example, if $S$ is a subspace, then for any plant $P$ satisfying these assumptions the only possible form of $C$ when it is convex is that $C$ is affine; it cannot, for example, be a ball.

\section{Non-affine example}

In the results of Section III-A, as well as in the first example of this section, the set of achievable closed loop 
maps is affine whenever it is convex. In this example, we show that more complicated sets are achievable as well. Consider the Banach space of real matrices under the standard induced 2-norm. Define the matrices

$$
P_{11}=\left[\begin{array}{l}
0 \\
0
\end{array}\right] \quad G=\left[\begin{array}{cccc}
0 & 1 & 0 & 0 \\
-1 & 0 & 1 & 0 \\
0 & 0 & 0 & 1 \\
1 & 0 & -1 & 0
\end{array}\right] \quad K=\left[\begin{array}{cccc}
t & 0 & 0 & 0 \\
0 & t & 0 & 0 \\
0 & 0 & s & 0 \\
0 & 0 & 0 & s
\end{array}\right]
$$

Here, the subspace of admissible controllers $S$ is the set of all $K$ above parameterized by $(t, s) \in \mathbb{R}^{2}$. Now consider two possible pairs of values for $P_{12}$ and $P_{21}$, and their corresponding sets of achievable closed-loop maps $C=$ $P_{11}-P_{12} h(K) P_{21}$.

(a) $P_{12}=\left[\begin{array}{cccc}0 & 2 & 0 & 0 \\ 0 & 0 & 0 & -2\end{array}\right]$ and $P_{21}=\left[\begin{array}{c}0 \\ 1 \\ 0 \\ -1\end{array}\right]$, which leads to $C=\left[\begin{array}{c}\frac{2 s}{1+s^{2}+t^{2}} \\ \frac{2 t}{1+s^{2}+t^{2}}\end{array}\right]$.

(b) $P_{12}=\left[\begin{array}{cccc}1 & 0 & 0 & 0 \\ 0 & 0 & -1 & 0\end{array}\right]$ and $P_{21}=\left[\begin{array}{c}0 \\ 2 \\ 0 \\ -1\end{array}\right]$, which leads to $C=\left[\begin{array}{c}\frac{\left(s^{2}+2\right) t^{2}}{1+s^{2}+t^{2}} \\ \frac{s^{2}\left(1-t^{2}\right)}{1+s^{2}+t^{2}}\end{array}\right]$.

As we vary $(s, t) \in \mathbb{R}^{2}$, one may check that $C$ is the unit disc in Case (a), and a more complicated nonconvex shape in Case (b). Figure 3 shows the sets $C$ in each case.

Both cases above have the same $G$ and $S$. It is straightforward to check that $S$ is not quadratically invariant under $G$. Therefore, Corollary 8 implies that $h(S)$ is not a convex set. Unfortunately, we cannot apply Corollary 9 to deduce anything about the convexity of $C$ because in both cases, $P_{12}$ is not left-invertible and $P_{21}$ is not rightinvertible. Indeed, we have shown that both a convex set (a) and a nonconvex set (b) are achievable depending on the choice of $P_{12}$ and $P_{21}$.

This example shows that in general, the convexity of $C$ does not depend on $S$ and $G$ alone, but also on $P_{12}$ and $P_{21}$. This idea is further discussed in [6], where $P_{12}$ and $P_{21}$ are used as part of a sufficient condition for convexity that is more general than quadratic invariance.

\section{Affine example}

We now show an example where the set of closed-loop maps is a convex set, and in particular is affine, even though $S$ is not quadratically invariant under $G$. This

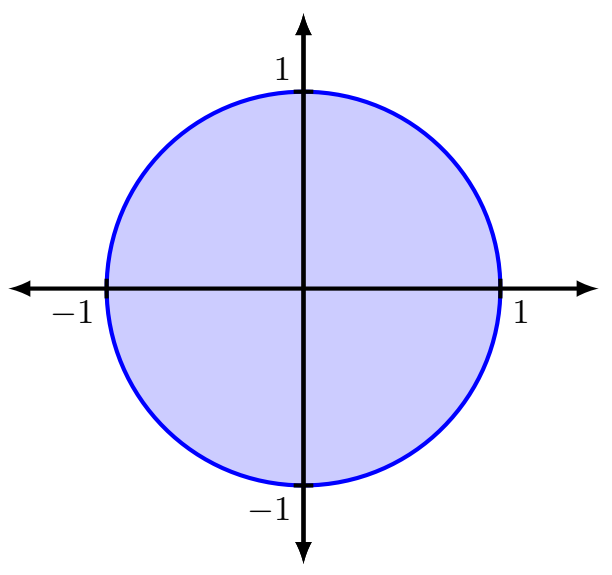

(a) first case, $C$ is convex.

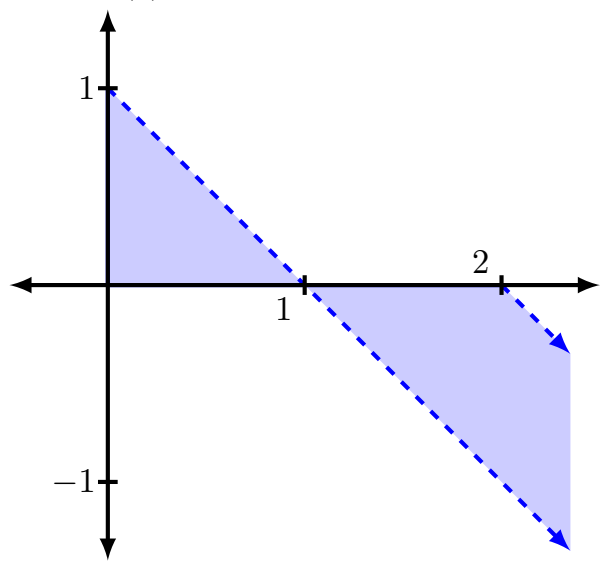

(b) second case, $C$ is nonconvex.

Figure 3: The set of achievable closed-loop maps described by both cases above. Solid lines are included in the set, while dashed lines are not.

example is a variant on an example shown in [5]. Suppose

$P=\left[\begin{array}{c:ccc}a & b_{1} & b_{2} & b_{2} \\ \hdashline c_{1} & g_{1} & 0 & 0 \\ c_{1} & g_{1} & 0 & 0 \\ c_{2} & g_{2} & g_{3} & g_{3}\end{array}\right] \quad S=\left\{\left[\begin{array}{ccc}k_{1} & 0 & 0 \\ 0 & k_{2} & 0 \\ 0 & 0 & k_{3}\end{array}\right] \mid k_{i} \in \mathbb{R}\right\}$

Here $a, b_{i}, c_{i}$ and $g_{i}$ are real numbers for simplicity, although it is straightforward to construct similar examples over the rational transfer matrices. The matrix $P$ is partitioned into its four blocks as shown with $P_{22}=G$; the dashed lines do not denote a state-space representation. The information constraint $S$ is the subspace of controllers with a diagonal structure. Note that $K G K$ is not diagonal for all diagonal $K$, so $S$ is not quadratically invariant under $G$. We will show that nonetheless that the set of achievable closed-loop maps $C$ is affine. To see why, define

$$
\tilde{P}=\left[\begin{array}{c:cc}
a & b_{1} & b_{2} \\
\hdashline c_{1} & g_{1} & 0 \\
c_{2} & g_{2} & g_{3}
\end{array}\right] \quad \tilde{S}=\left\{\left[\begin{array}{cc}
k_{1} & 0 \\
k_{2} & k_{3}
\end{array}\right] \mid k_{i} \in \mathbb{R}\right\}
$$


Here $\tilde{S}$ is clearly quadratically invariant under $\tilde{G}$. Therefore the set of achievable closed-loop maps $\tilde{C}$ is affine. It is straightforward to check that $C=\tilde{C}$. In fact, the parameterizations are exactly equal provided the $k_{i}$ are the same. So $C$ is affine despite $S$ not being quadratically invariant under $G$. Such transformations $(P, S) \mapsto(\tilde{P}, \tilde{S})$ are explored in [5].

\section{Conclusion}

It was previously known that when the set of decentralized controllers is a subspace $S$, quadratic invariance is a necessary and sufficient condition under which $h(S)=S$. In this paper, we showed that quadratic invariance is in fact necessary and sufficient for $h(S)$ to be convex. Furthermore, we showed that under certain invertibility conditions, quadratic invariance is also necessary and sufficient for the convexity of $C$, the set of achievable closed-loop maps.

This work therefore strengthens the utility of quadratic invariance as an indicator for tractability of decentralized control synthesis problems. However, there remains a nontrivial case, that when $S$ is not quadratically invariant and the aforementioned invertibility conditions do not hold. In that case one cannot draw any conclusions about the convexity of $C$. This was illustrated in Section IV, where we gave an example of a problem that is not quadratically invariant, but $C$ can be either convex or nonconvex, depending on the choice of the system parameters.

\section{References}

[1] A. A. Ahmadi and P. A. Parrilo. A complete characterization of the gap between convexity and SOS-convexity. SIAM Journal on Optimization, 23(2):811-833, 2013.

[2] V. D. Blondel and J. N. Tsitsiklis. A survey of computational complexity results in systems and control. Automatica, 36(9):1249-1274, 2000.

[3] Y.-C. Ho and K.-C. Chu. Team decision theory and information structures in optimal control problems - Part I. IEEE Transactions on Automatic Control, 17(1):15-22, 1972.

[4] L. Lessard. Tractability of complex control systems. PhD thesis, Stanford University, 2011.

[5] L. Lessard and S. Lall. Reduction of decentralized control problems to tractable representations. In IEEE Conference on Decision and Control, pages 1621-1626, 2009.

[6] L. Lessard and S. Lall. Internal quadratic invariance and decentralized control. In American Control Conference, pages 5596-5601, 2010.
[7] L. Lessard and S. Lall. Quadratic invariance is necessary and sufficient for convexity. In American Control Conference, pages 5360-5362, 2011.

[8] F. Lin, M. Fardad, and M. R. Jovanovic. Augmented Lagrangian approach to design of structured optimal state feedback gains. IEEE Transactions on Automatic Control, 56(12):2923-2929, 2011.

[9] A. Nayyar, A. Mahajan, and D. Teneketzis. Decentralized stochastic control with partial history sharing: A common information approach. IEEE Transactions on Automatic Control, 58(7):16441658, 2013.

[10] X. Qi, M. V. Salapaka, P. G. Voulgaris, and M. Khammash. Structured optimal and robust control with multiple criteria: A convex solution. IEEE Transactions on Automatic Control, 49(10):16231640, 2004.

[11] R. Radner. Team decision problems. The Annals of Mathematical Statistics, 33(3):857-881, 1962.

[12] M. Rotkowitz and S. Lall. Decentralized control information structures preserved under feedback. In IEEE Conference on Decision and Control, pages 569-575, 2002.

[13] M. Rotkowitz and S. Lall. A characterization of convex problems in decentralized control. IEEE Transactions on Automatic Control, 51(2):274-286, 2006.

[14] Ș. Sabău and N. Martins. Necessary and sufficient conditions for stabilizability subject to quadratic invariance. In IEEE Conference on Decision and Control, pages 2459-2466, 2011.

[15] N. Sandell, Jr. and M. Athans. Solution of some nonclassical LQG stochastic decision problems. IEEE Transactions on Automatic Control, 19(2):108-116, 1974.

[16] H. Shin and S. Lall. Decentralized control via Groebner bases and variable elimination. IEEE Transactions on Automatic Control, 57(4):1030-1035, 2012.

[17] T. Tanaka and C. Langbort. The bounded real lemma for internally positive systems and H-infinity structured static state feedback. IEEE Transactions on Automatic Control, 56(9):2218-2223, 2011.

[18] H. S. Witsenhausen. A counterexample in stochastic optimum control. SIAM Journal on Control, 6(1):131-147, 1968.

[19] K. Zhou, J. Doyle, and K. Glover. Robust and optimal control. Prentice-Hall, 1995.

[20] R. J. Zimmer. Essential results of functional analysis. University of Chicago Press, 1990. 